\title{
MICROBUNDLES AND BUNDLES
}

\author{
BY PER HOLM ${ }^{1}$
}

Communicated by S. Smale, November 9, 1965

The following concerns a generalization of the Kister-Mazur representation theorem for microbundles, which says that any microbundle over a locally finite, finite dimensional simplicial complex contains a bundle, unique up to bundle isomorphism. More precisely, the purpose of this note is to prove the following:

MiCRobundLE REPRESENTATION THEOREM. (a) Let $\mu: X \rightarrow^{\bullet} E \rightarrow^{p} X$ be an $R^{a}$-microbundle over a paracompact base space, and let $U \subset X$ be a neighbourhood of a closed set $A \subset X$. Suppose $\mu \mid U$ is actually an $R^{a-b u n d l e . ~ T h e n ~ t h e r e ~ i s ~ a ~ n e i g h b o u r h o o d ~} E^{\prime}$ of $s X$ in $E$ and a neighbour-

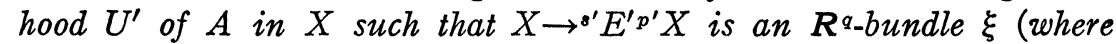
$i \circ s^{\prime}=s, p^{\prime} \circ i=p, i: E^{\prime}(E)$ and $\xi\left|U^{\prime}=\mu\right| U^{\prime}$.

(b) Suppose $\xi_{1}, \xi_{2}$ are $R^{a-b u n d l e s ~ c o n t a i n e d ~ i n ~} \mu$ and that $\xi_{1} \mid U^{\prime}$ $=\xi_{2} \mid U^{\prime}$ for some neighbourhood $U^{\prime}$ of $A$ in $X$. Then there is a bundle isomorphism $\xi_{1} \approx \xi_{2}$ which is the identity over $A$.

A proof of the representation theorem will be outlined after some preparatory work. It depends strongly on the germ extension theorem for trivial bundles (Theorem 1 below). This result is stated in Mazur [3] but seems false unless some restrictions are placed on the base space (or the germ). In the case where $X$ is paracompact it seems to follow from the general theory of dilation neighbourhoods as developed in [3]. In any case a direct proof is indicated below. It uses methods of Kister and Mazur generalized from the case where $X$ is a simplex to the case where $X$ is any topological space. Reportedly Mazur has used his theory of dilation neighbourhoods to establish the representation theorem in the case where $X$ is locally compact, normal and Lindelöf. Since any such space is of course paracompact his result is contained in ours.

The main results of this paper can be generalized to the case of numerable microbundles; cf. [1]. A more general and detailed version will appear elsewhere.

Finally the author wishes to thank Professor M. Hirsch and Professor E. Spanier for many enlightening discussions on the subject.

1. In the sequel we use the concepts and notations of Milnor [4] except for the following modifications. Instead of an isomorphism-

1 This work was written in Berkeley, California in spring 1965 while the author was supported by NAVF (Norway) and NSF (Grant GP-2497). 
germ $\mu \Rightarrow \mu^{\prime}$ of microbundles, we speak of an isogerm or, if $\mu$ equals $\mu^{\prime}$, of an autogerm. An embedding $\xi \rightarrow \xi^{\prime}$ of $R^{q}$-bundles with base $X$ is a bundle map (i.e. a map of total spaces preserving zero-sections and fibers) which is an open embedding. If it is onto, it is an isomorphism or an automorphism. Throughout this paper all base spaces of bundles and microbundles are paracompact.

We now consider autogerms and embeddings of the standard trivial $R^{q}$-bundle over some paracompact (Hausdorff) space $X$. In the case where $X$ is a point any autogerm $\varphi$ (of $\left(R^{q}, 0\right)$ ) is clearly represented by an embedding $\phi$. The image $\phi D_{1}$ of the closed unit ball at the origin in $R^{q}$ is a topological ball which is a neighbourhood of the origin 0 , hence contains a ball $D_{t}$ and is contained in a ball $D_{t^{\prime}}$. Following up $\phi$ by some suitable expansion changes $\phi$ into an embedding $\phi_{1}$ with germ $\varphi$ such that $\phi_{1} D_{1} \supset D_{2}$. Proceeding inductively one constructs embeddings $\phi_{2}, \phi_{3}, \cdots$ such that $\phi_{i} D_{i} \supset D_{i+1}$ and $\phi_{i} \mid D_{i-1}$ $=\phi_{i-1} \mid D_{i-1}, i=2,3, \cdots$. Then $\lim \phi_{i}$ is an automorphism of $R^{q}$ (i.e. a homeomorphism leaving the origin fixed) whose germ is $\varphi$. Thus any autogerm of the (trivial) $R^{a}$-bundle over a point is actually represented by an automorphism. The conclusion extends easily to trivial $R^{a}$-bundles over compact base spaces $X$ because of the fact that $\phi\left(X \times D_{1}\right)$ contains a neighbourhood $X \times D_{t}$ of the zero-section and is contained in some $X \times D_{t^{\prime}}$. The latter is generally not true for paracompact $X$. In this case, however, one verifies at least that any autogerm $\varphi: X \times R^{q} \Rightarrow X \times R^{q}$ can be represented by an embedding $\phi: X \times R^{q} \rightarrow X \times R^{q}$, and (less easily) that $\phi\left(X \times D_{1}\right)$ contains a diskbundle neighbourhood of the zero-section of varying cross-section and is contained in another one, where the radii in the fibers are measured by positive continuous functions on $X$. (These functions do not depend on the paracompactness of $X$.) One then proceeds in principle as before. Thus we get:

(1) THEOREM. Let $\varphi: X \times R^{q} \Rightarrow X \times R^{a}$ be an autogerm of the trivial $R^{a}$-bundle. Then there is an automorphism $\phi: X \times R^{a} \rightarrow X \times R^{q}$ whose germ is $\varphi$.

For any $R^{q}$-bundle $\xi: X \rightarrow^{8} E \rightarrow^{p} X$ if $A \subset X$, let $\xi\left|A: A \rightarrow^{8^{\prime}} E\right| A \rightarrow^{p^{\prime}} A$ denote the restriction of $\xi$ to $A$. The subset $A \subset X$ is trivializing for $\xi$ in case $\xi \mid A$ is isomorphic to the standard trivial $R^{q}$-bundle over $A$. Such an isomorphism is called a trivialization of $\xi$ over $A$ (or just a trivialization if $A=X$ ) and written $E \mid A \approx A \times R^{q}$. A partition of unity on $X\left(\pi_{i}, W_{i}\right), W_{i}=\pi_{i}^{-1}(0,1)$, is trivializing for $\xi$ in case each $W_{i}$ of the open cover $\left(W_{i}\right)$ of $X$ is trivializing. Any open cover of $X$ admits subordinate trivializing partitions of unity (since $X$ is paracompact), cf. Dold [1]. 
(2) Lemma. Let $\xi: X \rightarrow^{s} E \rightarrow^{p} X$ be an $R^{q}$-bundle and let $\varphi: E \Rightarrow X \times R^{q}$ be an isogerm of $\xi$ to the trivial $R^{q_{-} b u n d l e . ~ S u p p o s e ~ t h e r e ~ e x i s t s ~} a$ trivializing partition of unity $\left(\pi_{i}, W_{i}\right)_{i=1,2}$ for $\xi$ and trivialization $\phi_{1}: E \mid W_{1} \approx W_{1} \times R^{q}$ of $\xi$ over $W_{1}$ whose germ is $\varphi \mid\left(E \mid W_{1}\right)$. Then there exists a trivialization $\phi: E \approx X \times R^{q}$ of $\xi$ whose germ is $\varphi$ and such that $\phi\left|\left(E \mid W_{1}-W_{2}\right)=\phi_{1}\right|\left(E \mid W_{1}-W_{2}\right)$.

Lemma 2 is the key step in all the inductive arguments needed for the proof of the representation theorem. It is a refinement of the germ extension theorem which conversely is used in proving it. A fairly easy consequence is the following:

(3) Corollary. Let $\xi: X \rightarrow^{8} E \rightarrow^{p} X$ be an $R^{q}$-bundle and $\varphi: E \Rightarrow X \times R^{q}$ an isogerm to the trivial $\boldsymbol{R}^{a}$-bundle. Then there exists a trivialization $\phi: E \approx X \times R^{q}$ of $\xi$ whose germ is $\varphi$.

We are now ready to sketch the proof of the representation theorem: Let $\mu: X \rightarrow^{8} E \rightarrow^{p} X$ be a given $R^{a-m i c r o b u n d l e ~ w i t h ~ t r i v i a l i z i n g ~}$ partition of unity $\left(\pi_{i}, W_{i}\right)_{i \in J}$. For any subset $K \subset J$, let $W_{K}=\cup_{j \in K} W_{j}$ and $\pi_{K}=\sum_{i \in K} \pi_{i}$, so that $W_{k}=\pi_{K}^{-1}(0,1]$. Consider the collection of all triples $\left(K, \xi,\left(\phi_{i}\right)\right)$, where $K \subset J, \xi: W_{K} \rightarrow^{{ }^{s} K} E_{K} \rightarrow^{p K} W_{K}$ is an $R^{q_{-}}$ bundle contained in $\mu \mid W_{K}$ (i.e. $E_{K}$ is an open neighbourhood of $s W_{K}$ in $p^{-1} W_{K}$ with $s_{K}$ and $p_{K}$ induced from $s$ and $\left.p\right)$, and $\left(\phi_{i}\right)=\left(\phi_{i}\right)_{i \in K}$ is a family of trivializations $\phi_{i}: E_{K} \mid W_{i} \approx W_{i} \times R^{a}$ for $i \in K$. In this nonempty collection introduce an order relation $\leqq$ by defining $\left(K, \xi,\left(\phi_{i}\right)\right)$ $\leqq\left(K^{\prime}, \xi^{\prime},\left(\phi_{j}^{\prime}\right)\right)$ whenever the following is true:

(a) $K \subset K^{\prime}$,

(b) $x \in W_{K} \& \pi_{K}(x)=\pi_{K^{\prime}}(x) \Rightarrow p_{K}^{-1} x=p_{K^{\prime}}^{-1} x$,

(c) $e \in E_{K} \& \pi_{K} p(e)=\pi_{K^{\prime}} p(e) \Rightarrow \phi_{i}(e)=\phi_{i}^{\prime}(e)$ for any $i \in K$ such that $p(e) \in W_{i}$.

This order relation is in fact inductive and so each triple is contained in a maximal one. Finally notice that if $\mu, U$ and $A$ are as described under (a) in the representation theorem, then there exists a trivializing partition of unity $\left(\pi_{i}, W_{i}\right)_{i \in J}$ for $\mu$, a neighbourhood $U^{\prime}$ of $A$ in $U$ and a $K \subset J$ with $W_{K} \subset U$ such that if $i \in J-K$, then $W_{i} \cap U^{\prime}=\varnothing$. Thus there is a maximal triple majorizing $\left(K, \mu \mid W_{K}\right.$, $\left.\left(\phi_{i}\right)\right)$, say $\left(K^{\prime}, \xi,\left(\Psi_{i^{\prime}}\right)\right)$, and by definition of the ordering $\xi\left|U^{\prime}=\mu\right| U^{\prime}$. Moreover, since $\left(K^{\prime}, \xi,\left(\Psi_{i^{\prime}}\right)\right)$ is maximal, we have $K^{\prime}=J$, since otherwise it would be possible to enlarge $\left(K^{\prime}, \xi,\left(\Psi_{i^{\prime}}\right)\right)$ by gluing on some $(j, \eta, \Psi)$ for $j \in J-K^{\prime}$, using (3). This gives part (a) in the representation theorem. Part (b) actually follows from (a). In fact the condi-

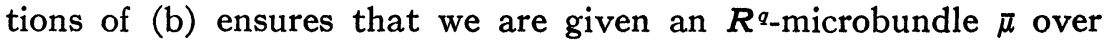
$X \times I$ which restricts to a bundle over some neighbourhood of $(X \times 0) \cup(A \times I) \cup(X \times 1)$ and such that $\bar{\mu}\left|X \times 0=\xi_{1}, \quad \bar{\mu}\right| X \times 1=\xi_{2}$ 
and $\bar{\mu}\left|(A \times I)=\xi_{1}\right| A \times I=\xi_{2} \mid A \times I$. By (a) there is a bundle $\bar{\xi}$ over $X \times I$ with the same properties. This gives (b).

The same techniques also give the following:

(4) Theorem. Let $\xi: X \rightarrow^{8} E \rightarrow^{p} X$ be an $R^{q}$-bundle over a paracompact base space $X$. Then there is a fiber homotopy $H: \operatorname{id}_{E} \simeq \operatorname{sp} \operatorname{rel} s X$ such that for $t \neq 1 H \mid E \times t$ is a bundle embedding.

From this result and the representation theorem follows by an argument of Milnor [5].

(5) Corollary. Let $\mu: X \rightarrow^{8} E \rightarrow^{p} X, \nu: E \rightarrow^{t} E^{\prime} \rightarrow^{q} E$ be microbundles, $X$ paracompact. Then the composite microbundle $\mu \circ \nu$ is isomorphic to $\mu \oplus s^{*} \nu$. Similarly, for microbundles $\mu, \mu^{\prime}$ over $X, \mu \oplus \mu^{\prime}$ is isomorphic to $\mu \circ p^{*} \mu^{\prime}$.

If $\mu$ and $\nu$ are actually bundles, the composite, although a microbundle, need not be a bundle. By the representation theorem it does contain an essentially unique bundle, however, which could be called the composite bundle, and which is bundle isomorphic to the Whitney sum of bundles $\mu \oplus s^{*} \nu$. This is still true if the word "bundle" is replaced by "orthogonal bundle."

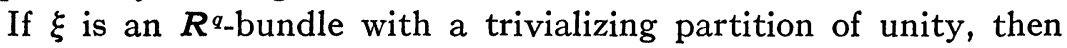
there is an associated $S^{a_{\text {-bundle }}} \xi_{\infty}$, determined up to natural isomorphism, with two sections $s_{0}, s_{\infty}$. The $R^{q_{\text {-bundle }}} \xi$ is contained in $\xi_{\infty}$ in such a way that the zero-section corresponds to $s_{0}$ and the total space $E$ to $E_{\infty}-$ im $s_{\infty}$. If $X$ is the base of $\xi$ (and $\xi_{\infty}$ ) and $A \subset X$ is any subspace, define the Thom space $T_{\xi}(X, A)$ to be the pointed space

$$
T_{\xi}(X, A)=E_{\infty} /\left(\mathrm{im} s_{\infty} \cup p_{\infty}^{-1} A\right),
$$

the collapsed subset im $s_{\infty} \cup_{p_{\infty}^{-1} A}$ serving as base point. By the representation theorem any microbundle over a paracompact base gets Thom spaces unique up to homeomorphism. In another note we use this concept to extend the Atiyah-Bott-Shapiro $S$-duality theorem to microbundles over topological manifolds.

\section{REFERENCES}

1. A. Dold, Partitions of unity in the theory of fibrations, Ann. of Math. (2) 78 (1963), 223-255. 199.

2. J. M. Kister, Microbundles are fibre bundles, Ann. of Math. (2) 80 (1964), 190-

3. B. Mazur, The method of infinite repetition in pure topology. I, Ann. of Math. (2) 80 (1964), 201-226.

4. J. Milnor, Microbundles. I, Topology 3 (1964), 53-80.

5. _- Piecewise linear microbundles (mimeographed notes).

University of California, Berkeley 